\title{
Tobramycin at subinhibitory concentration inhibits the Rhll/R quorum sensing system in a Pseudomonas aeruginosa environmental isolate
}

\author{
Fedora Babić ${ }^{1,2}$, Vittorio Venturi2 ${ }^{2 *}$ Gordana Maravić-Vlahoviček ${ }^{1 *}$
}

\begin{abstract}
Background: Antibiotics are not only small molecules with therapeutic activity in killing or inhibiting microbial growth, but can also act as signaling molecules affecting gene expression in bacterial communities. A few studies have demonstrated the effect of tobramycin as a signal molecule on gene expression at the transcriptional level and its effect on bacterial physiology and virulence. These have shown that subinhibitory concentrations (SICs) of tobramycin induce biofilm formation and enhance the capabilities of $P$. aeruginosa to colonize specific environments.
\end{abstract}

Methods: Environmental P. aeruginosa strain PUPa3 was grown in the presence of different concentrations of tobramycin and it was determined at which highest concentration SIC, growth, total protein levels and translation efficiency were not affected. At SIC it was then established if phenotypes related to cell-cell signaling known as quorum sensing were altered.

Results: In this study it was determined whether tobramycin sensing/response at SICs was affecting the two independent AHL QS systems in an environmental P. aeruginosa strain. It is reasonable to assume that $P$. aeruginosa encounters tobramycin in nature since it is produced by niche mate Streptomyces tenebrarius. It was established that SICs of tobramycin inhibited the RhII/R system by reducing levels of C4-HSL production. This effect was not due to a decrease of $r h / l$ transcription and required tobramycin-ribosome interaction.

Conclusions: Tobramycin signaling in P. aeruginosa occurs and different strains can have a different response. Understanding the tobramycin response by an environmental $P$. aeruginosa will highlight possible inter-species signalling taking place in nature and can possible also have important implications in the mode of utilization for human use of this very important antibiotic.

\section{Background}

Aminoglycoside antibiotics are clinically very important therapeutics widely used for treatment of chronic bacterial infections caused by Gram-negative bacteria. They bind to a specific domain of the prokaryotic $30 \mathrm{~S}$ ribosomal subunit and interfere with protein synthesis causing either inhibition of cell growth or cell death $[1,2]$. For example, tobramycin, an aminoglycoside produced by Streptomyces tenebrarius is commonly used for its

\footnotetext{
* Correspondence: venturi@icgeb.org; gordana@pharma.hr 'Department of Biochemistry and Molecular Biology, Faculty of Pharmacy and Biochemistry, University of Zagreb, Ante Kovačića 1, 10000 Zagreb, Croatia

${ }^{2}$ Bacteriology Group, International Centre for Genetic Engineering and Biotechnology, Area Science Park, Padriciano 99, 34149 Trieste, Italy
}

effectiveness against opportunistic cystic fibrosis (CF) infections of Pseudomonas aeruginosa [3]. Tobramycin affects protein synthesis by binding to the A site of the $30 \mathrm{~S}$ ribosomal subunit [4], interacting with the A1408 residue ( $E$. coli numbering) of the decoding site $16 \mathrm{~S}$ rRNA [5].

It has recently been reported, that antibiotics are not only small molecules with therapeutic activity in killing or inhibiting microbial growth, but can also act as signaling molecules affecting gene expression in bacterial communities [6-9]. Bacterial signaling molecules are defined as being able to (i) elicit a response unrelated to the metabolism or detoxification of the signaling molecule, (ii) accumulate extracellularly, (iii) generate a coordinated response once a critical concentration is 
reached. Antibiotics meet these criteria and now a few studies are beginning to lay the foundation that antibiotics can modulate global transcription also by affecting/ interfering with known global cascade responses [10-12]. This signaling activity occurs at much lower concentrations than those required for antibiosis being as low as $1 \%$ or as high as $50 \%$ of those required for inhibition (which is referred to as minimal inhibitory concentration or MIC) depending on the compound. The concentration at which antibiotic signaling is studied are in subinhibitory antibiotic concentrations, or SICs. SIC of antibiotics have been shown to affect several phenotypes in diverse bacteria through induced transcription modulation including virulence functions in group A streptococci [13], virulence and motility in Salmonella typhimurium [9] and biofilm formation in Pseudomonas aeruginosa [14].

A few studies have demonstrated the effect of tobramycin as a signal molecule on gene expression at the transcriptional level $[10,11]$ and its effect on bacterial physiology and virulence [14]. These studies have shown that low SICs of tobramycin induce biofilm formation [14] and enhance the capabilities of P. aeruginosa to colonize specific environments [11]. The response to SICs has therefore, important implications on the antibiotic therapy of $P$. aeruginosa infections, but the molecular mechanisms governing this response are currently unknown.

In $P$. aeruginosa, the regulation of many virulence associated factors, as well as biofilm formation, are under regulation of two hierarchically arranged $N$-acyl homoserine lactone (AHL) quorum-sensing (QS) systems, namely the LasI/R and RhII/R systems [3]. Each system contains one gene encoding AHL sensor/transcriptional regulator, i.e. $l a s R$ and $r h l R$, and a gene encoding an autoinducer synthase, i.e. lasI and rhlI, required for the synthesis of the autoinducer molecules $\mathrm{N}$-(3-oxo-dodecanoyl)-L-homoserine lactone (3-oxoC12-HSL) and N-(butanoyl)-L-homoserine lactone (C4HSL), respectively (reviewed by $[3,15,16]$ ). At high cell density in a situation of quorum AHL concentrations, 3-oxo-C12-HSL and C4-HSL interact directly with the cognate LasR and RhlR sensor/regulator affecting transcription of target genes.

Since QS is a global regulatory mechanism coordinated by bacterial communities, scientists have begun to investigate the possible link with antibiotic signalling. For example the response of $P$. aeruginosa to the macrolide antibiotic azithromycin studied by microarray, proteomics and phenotype analysis revealed that there is a large common subset of genes regulated by QS and azithromycin $[14,17,18]$. These studies demonstrated that azithromycin has considerable QS-antagonistic effects thus when applied it improves $P$. aeruginosa infections of CF patients. In fact, SIC of azithromycin were shown by microarray analysis to repress a large number of genes which are QS regulated and similar observations were made with other antibiotics [12]. In this study we were interested to determine whether tobramycin sensing/response at SICs was affecting the two AHL QS systems in $P$. aeruginosa. In this study we used an environmental isolate of $P$. aeruginosa PUPa3 in which the two AHL QS systems were not hierarchically organized. The reason for performing these studies using an environmental isolate of $P$. aeruginosa was because the producer of tobramycin, Streptomyces tenebrarius, lives in similar niches of $P$. aeruginosa hence it was of interest to determine if inter-species signalling could take place between them.

\section{Methods}

Bacterial strains, plasmids and growth conditions

Strains, plasmids and primers used in this study are listed in Table 1. P. aeruginosa PUPa3 has been previously isolated from the rice rhizosphere in India [19]. For DNA transformations E. coli DH5 $\alpha$ was used [20] while for triparental matings we used $E$. coli DH5 $\alpha$ (pRK2013) as helper [21]. Cultures were grown at $37^{\circ} \mathrm{C}$ aerobically in Luria-Bertani (LB) broth or $1.5 \%$ agar (Bacto-agar, BD Difco) plates containing appropriate antibiotics. P. putida was grown at $30^{\circ} \mathrm{C}$. Antibiotics were added when required at the following final concentrations: ampicillin $100 \mu \mathrm{g} / \mathrm{mL}$, tetracycline $15 \mu \mathrm{g} /$ $\mathrm{mL}$ (E. coli) or $100 \mu \mathrm{g} / \mathrm{mL}$ (Pseudomonas), gentamicin $100 \mu \mathrm{g} / \mathrm{mL}$ (Pseudomonas); kanamycin $100 \mu \mathrm{g} / \mathrm{mL}$, chloramphenicol $25 \mu \mathrm{g} / \mathrm{mL}$ (E. coli) or $250 \mu \mathrm{g} / \mathrm{mL}$ (Pseudomonas).

\section{DNA manipulations and plasmid constructs}

Recombinant DNA techniques, including digestion with restriction enzymes, agarose gel electrophoresis, purification of DNA fragments, ligation with T4 ligase and transformation of $E$. coli were performed as described [22]. Plasmids pLASI and pRHLI were constructed as follows. The lasI gene and rhlI gene promoters were amplified using $P$. aeruginosa PUPa3 chromosomal DNA as template and using primers lasI-fw/lasI-rv and rhlI-fw/rhlI-rv respectively, listed in Table 1. PCR fragments were ligated in pMOSBlue and digested with EcoRI and XbaI or EcoRI and KpnI respectively and then ligated in the lac $Z$ promoterless cloning vector pMP220, yielding pLASI and pRHLI respectively. The rhlR gene was amplified from PUPa3 chromosomal DNA and ligated into pGEM cloning vector, then digested with KpnI and HindIII and ligated into the corresponding sites of pQE30 generating pQERHLR. Methyltransferase genes $r m t A$ and $r m t C$ were kindly provided from Dr. Jason P. Rife (University of North 
Table 1 Strains, plasmids and primers used

\begin{tabular}{|c|c|c|}
\hline $\begin{array}{l}\text { Strains, Plasmids } \\
\text { and Primers }\end{array}$ & Characteristics & $\begin{array}{l}\text { Reference or } \\
\text { source }\end{array}$ \\
\hline \multicolumn{3}{|l|}{ Strains } \\
\hline \multicolumn{3}{|l|}{$\begin{array}{l}\text { Pseudomonas } \\
\text { aeruginosa }\end{array}$} \\
\hline PUPa3 & Wild type, rice rhizosphere isolate & [42] \\
\hline LASI & lasl::Km of P. aeruginosa PUPa3; Km & [43] \\
\hline RHLI & rhll::Gm of $P$. aeruginosa PUPa3; Gm ${ }^{r}$ & [43] \\
\hline DMI & lasl:::Km rhll::Gm of P. aeruginosa PUPa3; $\mathrm{Km}^{r} \mathrm{Gm}^{r}$ & [43] \\
\hline \multicolumn{3}{|c|}{ Pseudomonas putida } \\
\hline SM17 & rsal::TC of $P$. aeruginosa PUPa3; $T c^{r}$ & [43] \\
\hline \multicolumn{3}{|l|}{ E. coli } \\
\hline M15(pRep4) & $\begin{array}{l}\text { Derivative of } E \text {. coli } \mathrm{K}-12 \text {, containing pREP4 plasmid ensuring the production of high levels of lac } \\
\text { repressor protein; } \mathrm{Km}^{r}\end{array}$ & Qiagen \\
\hline $\mathrm{DH} 5 \alpha$ & F'lendA1 hsdR17 supE44 thi-1 recA1 gyrA relA1 (lacZYA-argF)U169 deoR [80dlac(lacZ)M15recA1] & [44] \\
\hline \multicolumn{3}{|l|}{ Plasmids } \\
\hline pMP220 & Promoter probe vector, IncP1; $T c^{r}$ & [45] \\
\hline pMOSBlue & Cloning vector; Ampr & $\begin{array}{l}\text { Amersham- } \\
\text { Pharmacia }\end{array}$ \\
\hline pGEM-T Easy & Cloning vector; Amp ${ }^{r}$ & Promega \\
\hline pBBRmcs3 & Broad-host-range vector; $T c^{r}$ & [46] \\
\hline pQE30 & Expression vector, $\mathrm{Amp}^{\mathrm{r}}$ & Qiagen \\
\hline pRTLF-1 & rpoS-lacZ translational fusion in pMP77; $\mathrm{Cm}^{r}$ & [47] \\
\hline pSB406 & contains a fusion of rhIR':.:IuxCDABE in pUC18; $\mathrm{Amp}^{r}$ & [48] \\
\hline pSB536 & ahy $^{+}$ahyl::/uxCDABE; ColE1 origin; $\mathrm{Amp}^{r}$ & [49] \\
\hline pRK2013 & $\mathrm{Tra}^{+} \mathrm{Mob}^{+}$ColE1 replicon; $\mathrm{Km}^{\mathrm{r}}$ & {$[50]$} \\
\hline pRPO220 & pMP220 containing promoter rpos; $T c^{r}$ & [51] \\
\hline pRSAL220 & pMP220 containing promoter rsaL; $T c^{r}$ & [47] \\
\hline pRHLI & pMP220 containing promoter $r h l l ; T c^{r}$ & This study \\
\hline pLASI & pMP220 containing promoter lasl; $T c^{r}$ & This study \\
\hline PQERHLR & pQE30 containing $r h / R ; A^{m} p^{r}$ & This study \\
\hline PRMTA & pBBR3 containing $r m t A ; T c^{r}$ & This study \\
\hline PRMTC & pBBR3 containing $r m t C ; T c^{r}$ & This study \\
\hline \multicolumn{3}{|l|}{ Primers } \\
\hline RhIR-fw & GGGGTACCAGGAATGACGGAGGCTIITTG & This study \\
\hline RhIR-rv & GAAGCTTGATGAGGCCCAGCGCCGCGG & This study \\
\hline rhll-fw & CCGAATTCCACCACAAGAACATCC & This study \\
\hline rhll-rv & ATGGTACCAGCGATTCAGAGAGCAA & This study \\
\hline lasl-fw & GGAATTCGGGCTGTGTTCTCTCGTGTG & This study \\
\hline las-rv & CTCTAGAGAACTCTTCGCGCCGACCAA & This study \\
\hline$R m t A-f w$ & GGGTACCAATAATTITGTTTAACTITA & [52] \\
\hline RmtA-rv & CCCTCGAGTCACTTATTCC & This study \\
\hline RmtC-fw & ATCTGCAGAATAATTTTGTTAACTITA & This study \\
\hline RmtC-rv & ATACTAGTTTACAATCTCGATACGAT & This study \\
\hline
\end{tabular}

$\mathrm{Ap}^{r}, \mathrm{Km}^{\mathrm{r}}, \mathrm{TC}^{\mathrm{r}}, \mathrm{Gm}^{\mathrm{r}}$, and $\mathrm{Cm}^{\mathrm{r}}$, resistant to ampicillin, kanamycin, tetracycline, gentamicin, and chloramphenicol, respectively

Carolina, USA) as expression vectors pET-15b(+) with coding genes for $r m t A$ and $r m t C$ respectively. The npmA gene synthesis was ordered from the Epoch Biolabs, INC. following the sequence published in [23]. The gene was subsequently cloned in pBBRmcs 3 with restriction sites XbaI and SacI generating pNPMA. $r m t A$ and $r m t C$ were cloned in pET-25b(+) vectors with restriction sites NdeI and XhoI after PCR amplification using primers RmtA-fw/RmtA-rv and RmtC-fw/RmtCrv respectively. PCR fragments were then ligated in pMOSBlue, digested with KpnI and XhoI or PstI and SpeI respectively and ligated in pBBRmcs3 generating pRMTA and pRMTC. All plasmid constructs were verified by DNA sequencing (Macrogen, Korea). 
Determination of Minimal Inhibitory Concentration (MIC) The minimal inhibitory concentration (MIC) of tobramycin (Sigma-Aldrich) for $P$. aeruginosa $\mathrm{PUPa} 3$ was determined in LB broth after 24 hours incubation at $37^{\circ} \mathrm{C}$ using the MIC dilution method as previously described [24]. Briefly, overnight cultures of PUPa3 were diluted 100 fold in fresh LB medium and grown until the $\mathrm{OD}_{600}$ of 1 . LB medium containing various concentrations of tobramycin $(0.05-320 \mu \mathrm{g} / \mathrm{mL})$ was inoculated with $10^{6}$ cells and grown in microtiter plates. MIC was determined as a minimal concentration of tobramycin that prevents the visible growth of bacteria.

\section{Determination of growth rate and of total cell protein and $\beta$-galactosidase assay}

Growth curves were performed in duplicates in LB or LB medium supplemented with various concentrations of tobramycin $(1 / 16,1 / 8,1 / 4,1 / 2$ of MIC). Overnight culture of $P$. aeruginosa $\mathrm{PUPa} 3$ was diluted 100 fold in fresh $\mathrm{LB}$ medium and cultures were grown at $37^{\circ} \mathrm{C}$ measured the $\mathrm{OD}_{600}$ every 20 minutes. $\beta$-galactosidase activities were determined by the Miller method [22] and levels of proteins were determined by the Bradford assay [25].

\section{Determination of swarming ability, proteolytic activity and biofilm formation}

Swarming assay was performed on $0.5 \%$ LB agar plates as previously described [26] supplemented with SIC of tobramycin (25\% of MIC). $1.5 \mu$ l of overnight culture of PUPa3 was inoculated on swarming plates and incubated at $37^{\circ} \mathrm{C}$ for 24 hours. Synthetic C4-HSL (purchased from Fluka-Sigma-Aldrich) was added in agar plates in final concentration of $2 \mu \mathrm{M}$. Proteolytic activity was determined in the appropriate indicator plates as previously reported [27] in presence or absence of $25 \%$ of $\mathrm{MIC}$ of tobramycin and grown at $37^{\circ} \mathrm{C}$ after 24 hours.

Biofilm formation was quantified by measuring $\mathrm{A}_{570}$ of crystal-violet staining of adherent cells. $2 \mathrm{ml}$ of overnight culture from $P$. aeruginosa $\mathrm{PUPa} 3$ grown in $\mathrm{AB}$ minimal medium was centrifuged and pellet was washed in fresh media. New inoculum of $\mathrm{OD}_{600}$ of 0,01 was grown at $37^{\circ} \mathrm{C}$ for 24 hours in wells of 96 -well polystyrene microtiter plates (Nunc) using $100 \mu \mathrm{l}$ of culture per well (16 duplicates per condition per experiment). Planktonic cells were removed and the wells with adherent biofilms were stained using $0,1 \%$ crystal-violet for $30 \mathrm{~min}$ at room temperature, washed three times with water and dried. To solubilize adsorbed crystal violet, $150 \mu \mathrm{l}$ of DMSO was added in each well and incubated for 20 minutes. The absorbance was read at $570 \mathrm{~nm}$. Unpaired t-test and ANOVA test (Prisma 5.5 software, GraphPad Software, San Diego, California) was used to establish significance of differences between means ( $P$ value of $<0,005)$. MIC was determined for the planktonic cells in the culture used as the inoculum. MIC of tobramycin was $8 \mu \mathrm{g} / \mathrm{ml}$ and $25 \%$ of MIC was used as subinhibitory concentration.

\section{Extraction of AHL signal molecules}

For the extraction of the AHL molecules, $P$. aeruginosa PUPa3 was grown until $\mathrm{OD}_{600}$ reached 2 in $50 \mathrm{ml}$ of $\mathrm{LB}$ medium or LB medium supplemented with SIC of tobramycin, and supernatant of the culture was extracted with an equal volume of ethyl acetate acidified with $0.1 \%$ acetic acid. The extract was then dried and resuspended in a small volume of ethyl acetate as previously described [26].

\section{Determination of C4-HSL, of 3-oxo-C12-HSL and of pyocyanin levels}

C4-HSL levels were determined as follows. Bioluminescence assay was performed using biosensors specific for C4-HSL, i.e. E. coli pSB406 [28] and E. coli pSB536 [29]. Overnight liquid cultures of the two reporter strains were diluted 1:100 in LB and grown till $\mathrm{OD}_{600}$ of 1 then added to the wells of black clear-bottom 96-well plates (OptiPlate microtiter trays, Perkin-Elmer, USA) and supplemented with various concentrations of synthetic C4-HSL to generate standard curve or with various amounts of $P$. aeruginosa AHL extracts. Cultures were then grown at $37^{\circ} \mathrm{C}$ and $\mathrm{OD}_{600}$ and luminescence from each well were recorded in a Wallac EnVision 2104 multilabel reader (Perkin-Elmer). Relative light intensity of extracts of $P$. aeruginosa grown in the presence of tobramycin was defined as the percentage of emitted light (British light units) under these conditions compared with the mean value of $P$. aeruginosa extracts grown without antibiotic.

3-oxo-C12-HSL was determined as previously described [30]. Briefly, $\beta$-galacotsidase activities were measured using $P$. putida SM17 (pRSAL220) lacZ based reporter strain upon addition of PUPa3 AHL extracts. Overnight cultures of $P$. putida were diluted up in $5 \mathrm{ml}$ volume to an $\mathrm{OD}_{600}$ of 0.3 and $P$. aeruginosa $\mathrm{AHL}$ extracts were added. $\beta$-galactosidase activity was measured during various stages of growth. All assays were performed in triplicate.

Pyocyanin levels were determined as previously described [31]. Briefly, P. aeruginosa PUPa3 was grown with aeration at $37^{\circ} \mathrm{C}$ in $\mathrm{LB}$ broth. Each culture was inoculated from a fresh overnight culture adjusted an optical density at $600 \mathrm{~nm}$ of 0.02 before incubation. Quantification of pyocyanin production was done by extracting a $5 \mathrm{ml}$ of culture with $3 \mathrm{ml}$ of chloroform followed by mixing with $1 \mathrm{ml}$ of $0.2 \mathrm{M} \mathrm{HCl}$. The absorbance of upper red phase was measured at $520 \mathrm{~nm}$ and amount of pyocyanin was determined as previously described [31]. 


\section{Results}

MIC and SIC of tobramycin for $P$. aeruginosa PUPa3

The MIC of tobramycin for strain PUPa3 was determined to be $0.2 \mu \mathrm{g} / \mathrm{mL}$ in LB broth and $2 \mu \mathrm{g} / \mathrm{mL}$ on $\mathrm{LB}$ agar plates. We then established that $25 \%$ of the MIC of tobramycin (i.e. $0.05 \mu \mathrm{g} / \mathrm{mL}$ ) was subinhibitory (SIC), since (i) it did not affect the growth rate of $P$. aeruginosa PUPa3 in LB media (data not shown), (ii) did not alter the protein synthesis as determined by measurement of total cellular protein (data not shown) and (iii) it did not affect translation efficiency because did not alter $\beta$-galactosidase activity of PUPa3 harboring the rpoS-lacZ translational fusion at various growth stages (data not shown).

\section{SIC of tobramycin affects swarming motility, biofilm formation and pyocyanin production in $P$. aeruginosa PUPa3}

It was of interest to determine the impact of SIC of tobramycin on the AHL QS response in P. aeruginosa PUPa3 using swarming assays. It was previously established that in $P$. aeruginosa PUPa3 swarming is regulated by the RhlI/R AHL QS system [26]. We observed as expected that the rhlI and double mutant (lasI/rhlI) were impaired in swarming whereas strain PUPa3 swarmed well. We then tested swarming abilities at SIC and established that strain PUPa3 did not swarm under these conditions indicating that $0.05 \mu \mathrm{g} / \mathrm{mL}$ of tobramycin impaired swarming (Figure 1a). Importantly, swarming can then be rescued by adding exogenous synthetic C4-HSL, indicating that possibly the presence of SIC of tobramycin affected the production levels C4-HSL (Figure 1b). Biofilm formation was also reduced when $P$. aeruginosa strain $\mathrm{PUPa} 3$ grows under SIC of tobramycin; this reduction could then be rescued by the exogenous addition of C4-HSL (Figure 2). Another phenotype previously reported to be regulated by the RhlI/R system is the production of the toxic pyocyanin pigment [32]. We also assayed for pyocyanin production (Figure 3 ) and determined that (i) the rhlI mutant of $P$. aeruginosa $\mathrm{PUPa} 3$ no longer produced pyocyanin and (ii) SIC concentrations of tobramycin reduced pyocyanin production which could be also rescued by addition of exogenous C4-HSL (Figure 3). It was therefore concluded that pyocyanin in strain PUPa3 is regulated by the RhlI/R system and that tobramycin affected the production levels.

In order to establish whether SIC of tobramycin affected the LasI/R AHL QS system of strain PUPa3, we assayed for protease production since as previously determined, protease activity is regulated by LasI/R [26]. It was established that when $P$. aeruginosa was grown in the presence of SIC of tobramycin proteolytic activity was unaltered when compared to $P$. aeruginosa grown in the absence of the antibiotic (Figure 4). It was therefore concluded that the LasI/R system was not affected by SIC of tobramycin.

\section{P. aeruginosa PUPa3 grown in SIC of tobramycin lowers C4-HSL levels}

In order to establish whether SIC of tobramycin affected AHL levels we first assayed rhlI and lasI transcription via a rhlI-lacZ and lasI-lacZ transcriptional gene promoter fusions. $\beta$-galactosidase activity in strain PUPa3 harboring either the plasmid pRHLI or pLASI showed no significant differences at various stages of growth in presence of SIC of tobramycin when compared to PUPa3 grown without tobramycin (Figure 5). It was therefore concluded that SIC of tobramycin did not affect the transcription rates of the rhlI and lasI AHLs synthases. Levels of 3-oxo-C12-HSL from PUPa3 extracts were then measured using the $P$. putida SM17 (pRSAL220) specific 3-oxo-C12-HSL sensor and no significant difference has been detected between cultures of PUPa3 of different growth stages grown in presence or absence of $\mathrm{SIC}$ of tobramycin (Figure 6b). Two E. coli luxCDABE based biosensors specific to C4-HSL were used in order to establish C4-HSL levels. Bioluminescence assays demonstrated that a significant reduction (20 - 25\%) of C4-HSL was observed when $P$. aeruginosa PUPa3 grown in presence of SIC of tobramycin (Figure 6a). It was concluded that SIC of tobramycin did not affect 3-oxoC12-HSL levels but did lower C4-HSL levels.

\section{SIC of tobramycin response requires interaction with ribosome}

In order to determine the importance of tobramycinribosome interaction in the signaling role in lowering C4-HSL levels, the npmA, rmtA and $r m t C$ methyltransferase genes were cloned and expressed in $P$. aeruginosa PUPa3. These methyltransferases are known to specifically methylate nucleotides A1408 [23] and G1405 $[33,34]$ respectively, within the decoding A site in small ribosomal subunit which then block tobramycin-ribosome interaction rendering the bacterium resistant to tobramycin. As observed above, $P$. aeruginosa PUPa3 could not swarm in the presence of SIC of tobramycin but when either the $n p m A$ or $r m t A$ or $r m t C$ genes were expressed using plasmids pNPMA, pRMTA and pRMTC respectively, swarming was restored (Figure 7). It was concluded that the observed effect of SIC of tobramycin in affecting C4-HSL dependent swarming involves tobramycin-ribosome interaction.

\section{Discussion}

It is important for us bacteriologists to remember that most bacterial species in any given environment 


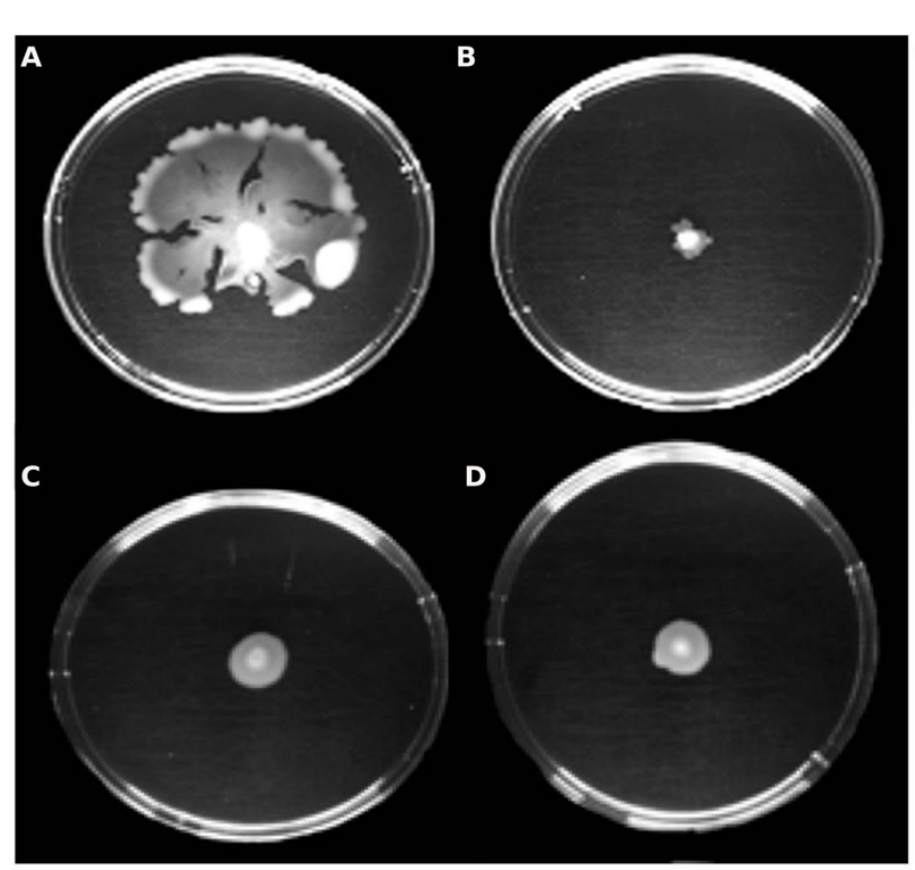

\section{A}

\section{B}
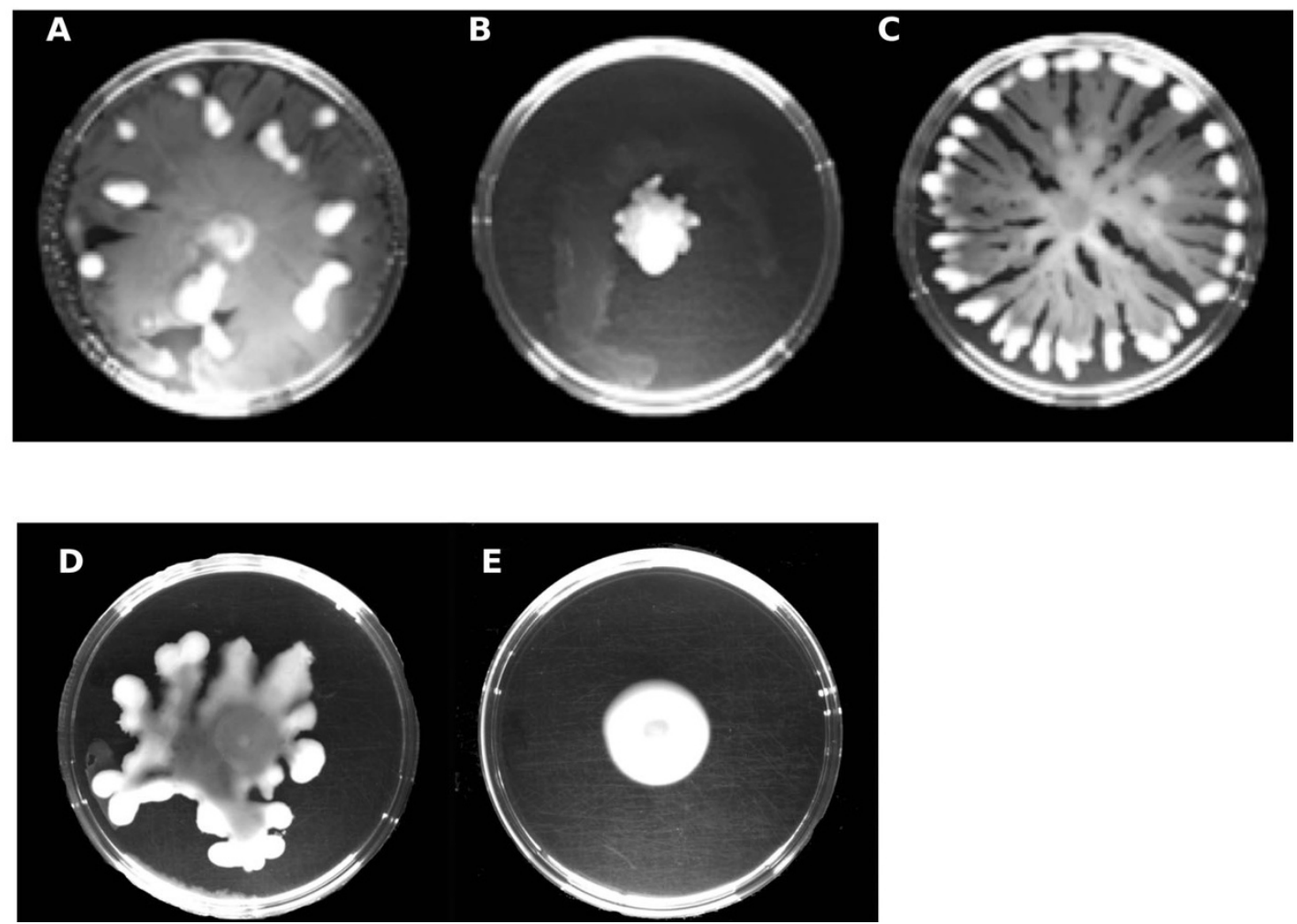

Figure 1 Effect of subinhibitory concentration of tobramycin on swarming motility in $P$. aeruginosa PUPa3. a) Swarming of $P$. aeruginosa PUPa3 on $0.5 \% \mathrm{LB}$ agar plates at $37^{\circ} \mathrm{C}$ after $24 \mathrm{hrs}$. WT swarms (A), but in presence of SIC of tobramycin $(0.05 \mu \mathrm{g} / \mathrm{mL}) \mathrm{cannnot} \mathrm{swarm} \mathrm{(B).}$

Swarming was not present in the rhll mutant (C) nor in double mutant las//rhll (D). b) Swarming of $P$. aeruginosa PUPa3 on $0.5 \%$ LB agar plates at $37^{\circ} \mathrm{C}$ after $40 \mathrm{hrs}$. Normal swarming occurs in WT (A), no swarming is present in WT on plate supplemented with SIC of tobramycin (B) and swarming was restored upon the addition of $2 \mu \mathrm{M}$ exogenous synthetic C4-HSL (C). Swarming in the rhll mutant was also restored upon the addition of $2 \mu \mathrm{M}$ exogenous synthetic C4-HSL (D), but not upon the addition of $2 \mu \mathrm{M}$ exogenous synthetic 3-oxo-C12-HSL (E). 


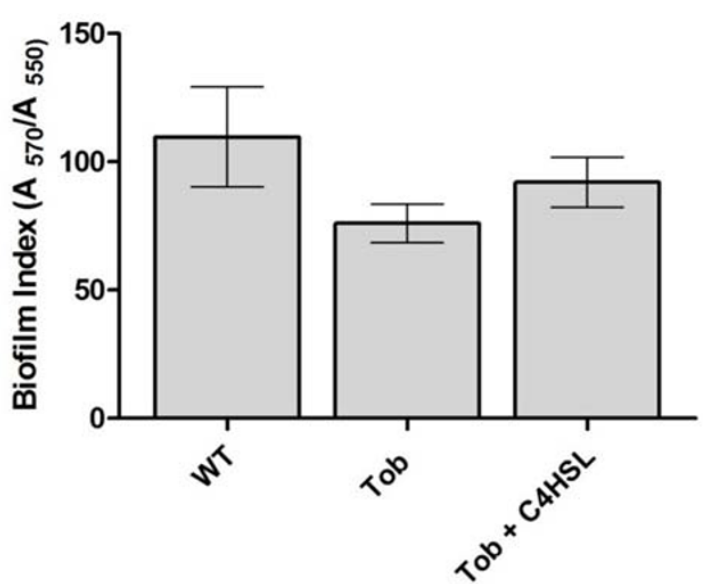

Figure 2 Effect of subinhibitory concentration of tobramycin on biofilm growth. Biofilm formation was reduced when PUPa3 was grown under subinhibitory concentration of tobramycin (Tob). Biofilm formation was rescued by adding $2 \mu \mathrm{M}$ exogenous C4-HSL $(T o b+C 4 H S L)$.

encounter a huge mixed population of prokaryotes meaning that bacteria do not live in isolation or in monoculture but in mixed communities. It is also most probable that in these mixed and diverse consortia there is considerable competition as well as constant communication. It is assumed that antibiotics with inhibitory

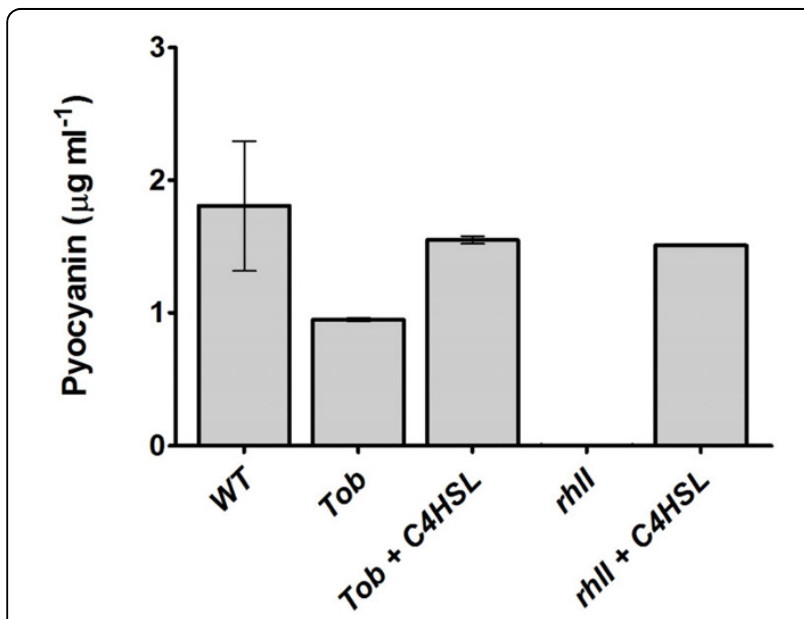

Figure 3 Pyocyanin production in PUPa3 in early stationary growth phase. All strains were grown in LB media. Presence of SIC of tobramycin in wild type significantly decreases levels of pyocyanin production $(P<0.05)$. Values shown in graph are means from three independent biological experiments, normalized to culture density $\left(\mathrm{OD}_{600}\right)$. WT, is strain PUPa3; Tob, is strain PUPa3 grown in the presence of SIC of tobramycin $(0.05 \mu \mathrm{g} / \mathrm{mL})$; Tob C4HSL, is strain PUPa3 grown in the presence of SIC of tobramycin $(0.05 \mu \mathrm{g} / \mathrm{mL})$ and $2 \mu \mathrm{M}$ of C4-HSL; rhll, is the rhll knock-out mutant of strain PUPa3; rhll C4HSL, is the rhll knock-out mutant of strain PUPa3 grown in the presence of exogenously added $2 \mu \mathrm{M}$ of C4-HSL.

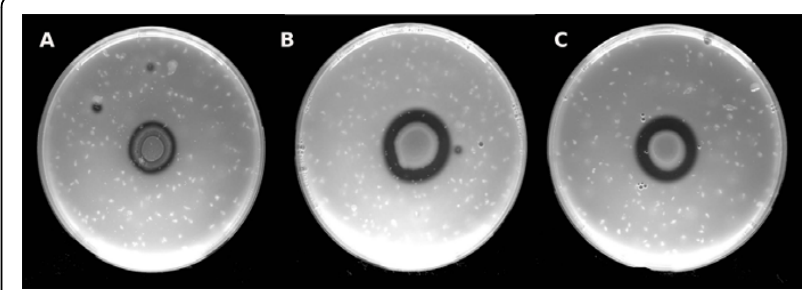

Figure 4 Proteolytic activities determined in the appropriate indicator LB plates. The $P$. aeruginosa las/ mutant $(A)$ showed fourfold reduced proteolytic activity compared to WT (B). WT shows no difference in proteolytic activity in the presence of $0.05 \mu \mathrm{g} / \mathrm{mL} \mathrm{SIC}$ of tobramycin (C).

activity are important as weapons in inter-microbial competition. In recent years however scientists are beginning to investigate that antibiotics have functional interactions with cellular processes which are different from the primary antibiotic inhibitory effect (reviewed by $[7,9])$. Several studies have now shown that subinhibitory concentrations (SICs; these do not affect growth and protein synthesis) of several antibiotics can transcriptionally modulate a large number of genes [10-12].

Here we began to study the effect of SICs of tobramycin on an environmental isolate of P. aeruginosa. Tobramycin is produced by Streptomyces tenebrarius which is a soil organism and therefore probably shares similar niches to $P$. aeruginosa since it is also found in the soil. $P$. aeruginosa in nature therefore encounters tobramycin and could have evolved the ability to respond to this molecule possibly allowing adaptation to co-exist or compete with $S$. tenebrarius. Importantly, $P$. aeruginosa possesses an inducible resistance to tobramycin [35] thus suggestive of a response and adaptive behavior towards the compound. Since $P$. aeruginosa possesses a major transcription response to AHL signals influencing the transcription of over $6 \%$ of the genome [16], it was of interest to determine if AHL QS was affected by SICs of tobramycin. The P. aeruginosa PUPa3 environmental isolate possesses the two AHL QS systems which act independently and are not hierarchically organized [26]. To our knowledge all other studies in $P$. aeruginosa involving antibiotics as signal molecules were performed using the clinical isolates. In fact it was previously reported that SIC of tobramycin in strain $P$. aeruginosa PAO1 (clinical isolate of 1950) also induces a reduction in C12-3-oxo-HSL and C4-HSL production [36]. It must be noted however that the two AHL systems in strain PAO1 are organized in a hierarchy; the Las system regulating in a cascade the rhl system [37]. SICs of tobramycin in strain PUPa3 reported here was found to lower only the C4-HSL levels by over $20 \%$ which was shown to be enough to inhibit swarming and lower pyocyanin levels which are both regulated by the 


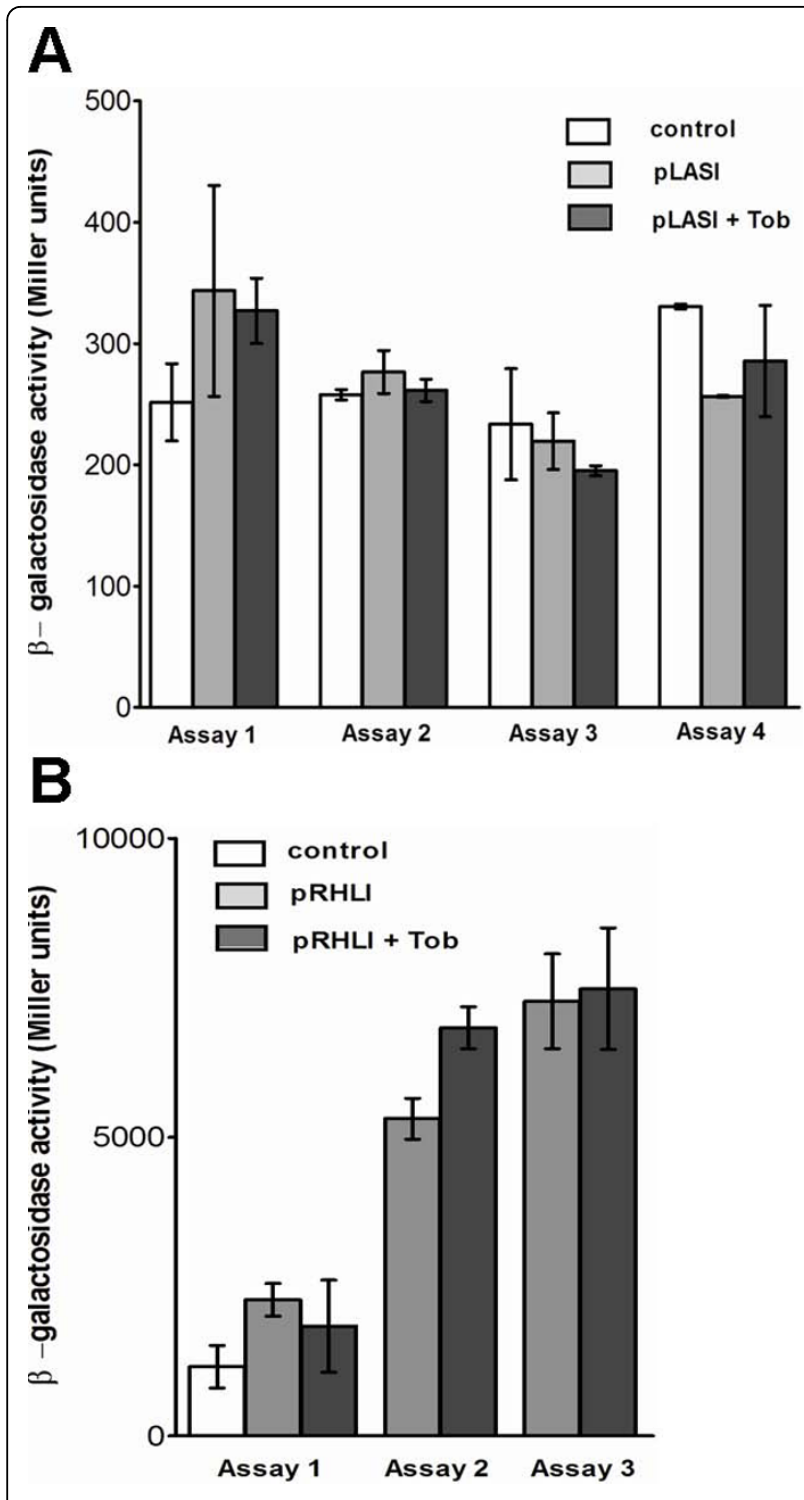

Figure 5 lasl and rhll gene promoter activities and quantitative determination of 3-oxo-C12-HSL. a)/as/ gene promoter activities measured via $\beta$-galactosidase assay in PUPa3 in different stages of growth. The white colorless bars represents the control culture P. aeruginosa PUPa3 with the vector PMP220; light grey represents $P$. aeruginosa PUPa3 harboring pLASI; dark grey, represents $P$. aeruginosa PUPa3 harboring PLASI with addition of SIC of tobramycin $(0.05 \mu \mathrm{g} / \mathrm{mL})$. b) rhll gene promoter activities measured via $\beta$-galactosidase assay in PUPa3 in different stages of growth. The white colorless bars represents the control culture $P$. aeruginosa PUPa3 with the vector pMP220; light grey represents $P$. aeruginosa PUPa3 harboring $\mathrm{pRHLI}$; dark grey, represents $P$. aeruginosa PUPa3 harboring PRHLI with addition of SIC of tobramycin $(0.05 \mu \mathrm{g} / \mathrm{mL})$. The $\beta$-galactosidase activities were performed at different stages of growth: assay 1 was performed during log phase, assay 2 during early stationary phase, assay 3 and assay 4 during stationary phase. Each assay was performed in biological triplicates. Error bars represent the standard deviations calculated from at least three separate experiments

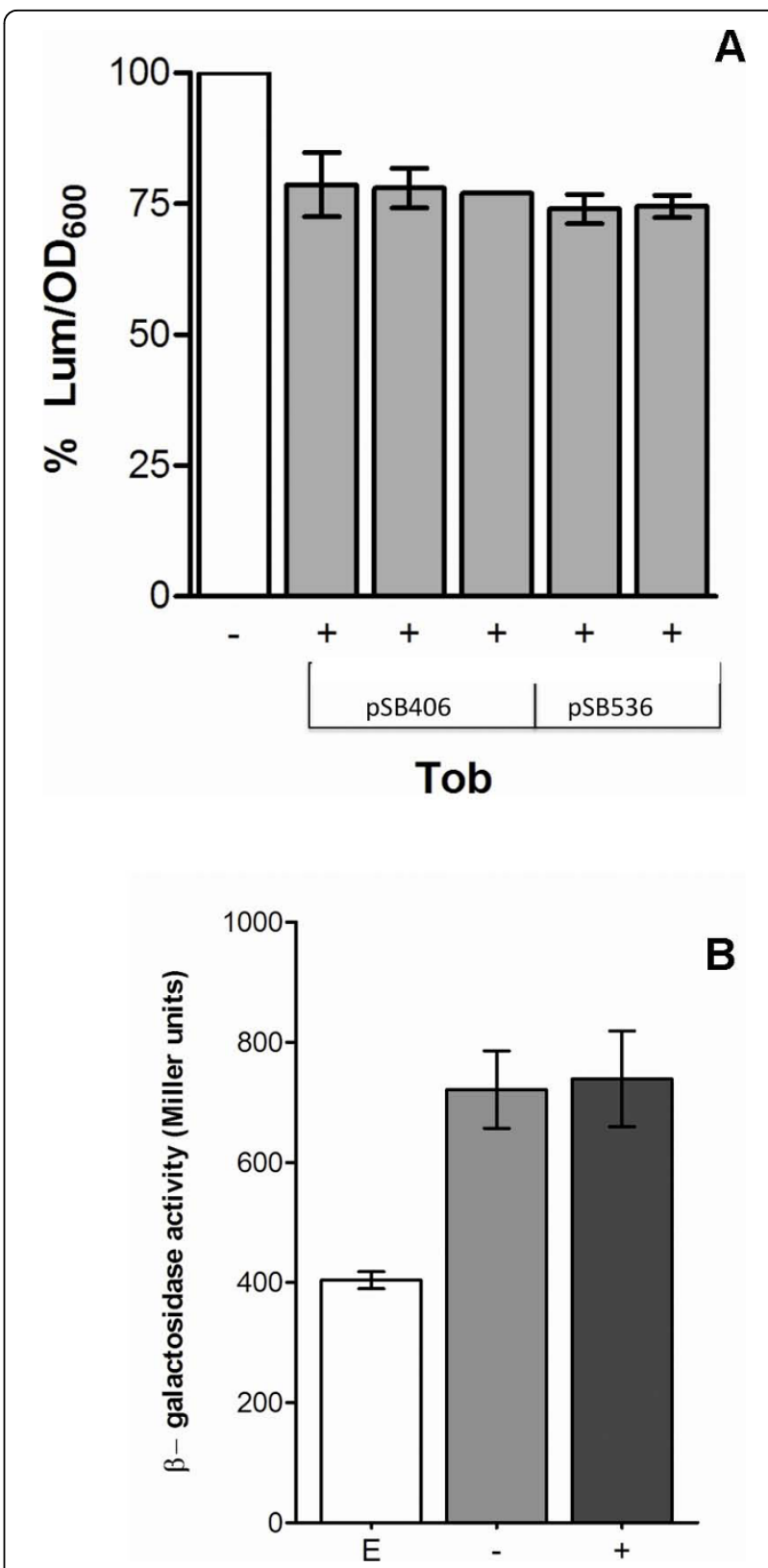

Figure 6 C4-HSL and 3-oxo-C12-HSL quantification. a) C4-HSL quantification using two E. coli luxCDABE specific C4-HSL biosensors harbored in pSB406 and pSB536. Bioluminescence induced by AHL extracts of PUPa3 corresponding to $7 \times 10^{9}$ cells grown in presence of SIC of tobramycin $(0.05 \mu \mathrm{g} / \mathrm{mL})$ at $37^{\circ} \mathrm{C}$ was defined as the percentage relative to the same strain without antibiotic (white bar). Each assay was an independent experiment measured in triplicates. +/- represents extract from PUPa3 grown in presence or absence of $\mathrm{SIC}$ of tobramycin added to biosensor culture. b) 3-oxo-C12-HSL quantification: the columns represent the 3-oxo-C12-HSL quantification determined by using the biosensor P. putida SM17 (pRSAL220). E represents $5 \mathrm{ml}$ of biosensor culture supplemented with $5 \mu$ l ethyl-acetate, $+/$ - addition of $5 \mu$ l of AHL extract of PUPa3 (equivalent to $9.2 \times 10^{9}$ cells) grown in presence or absence of SIC of tobramycin $(0.05 \mu \mathrm{g} / \mathrm{mL})$. 

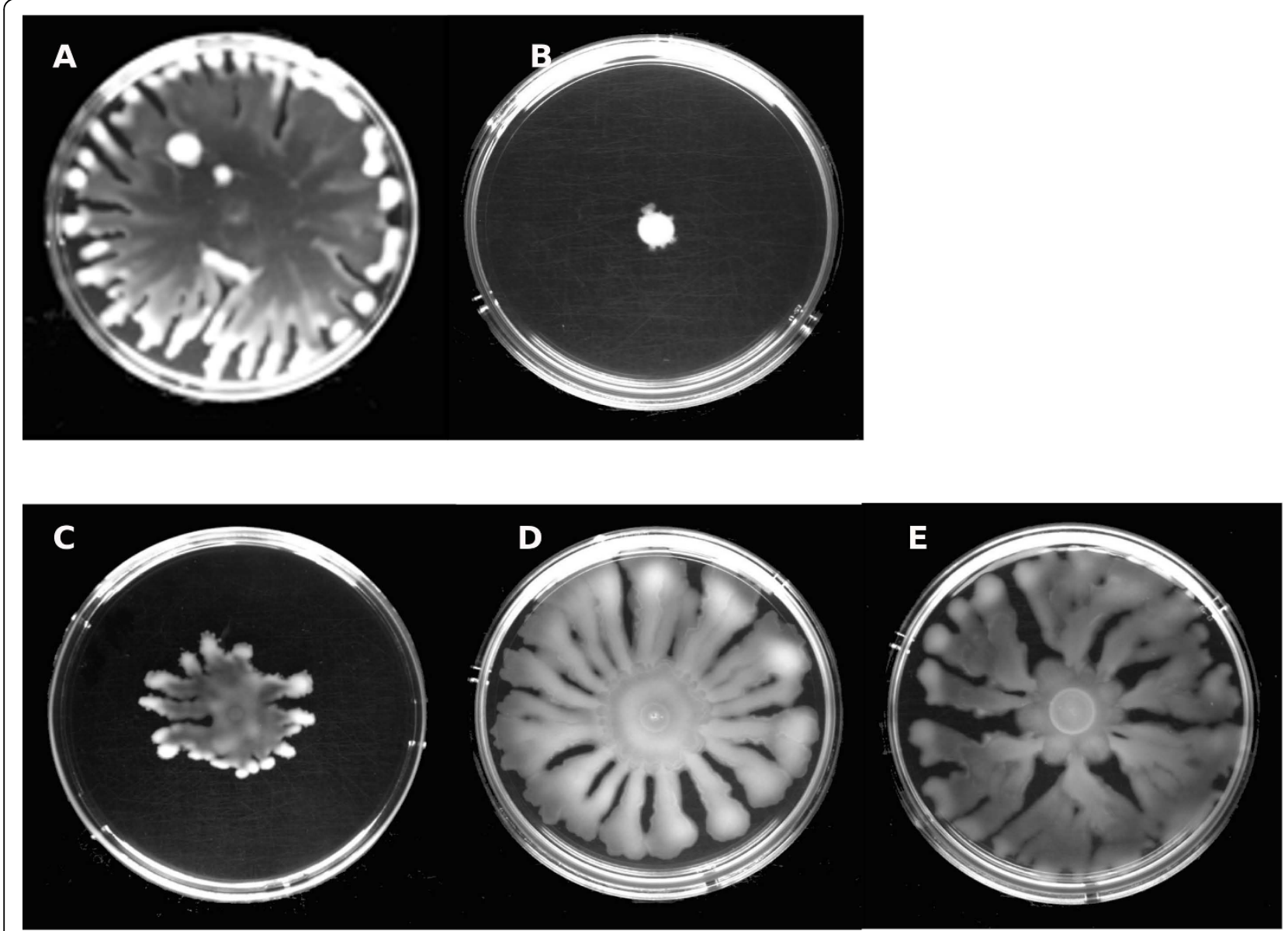

Figure 7 Swarming of $P$. aeruginosa PUPa3 on $\mathbf{0 . 5 \% ~ L B}$ agar plates at $37^{\circ} \mathrm{C}$ after $\mathbf{2 4} \mathrm{hrs}$. In the presence of SIC tobramycin $(0.05 \mu \mathrm{g} / \mathrm{mL})$ no swarming is present in WT (B), but the swarming was restored with the expression of RmtA (C), RmtC (D) or NpmA (E) methyltransferases as in LB plates inoculated with WT (A).

RhlI/R system. Importantly both of these effects were recovered upon addition of exogenous C4-HSL indicating that they were entirely due to interference with the RhlI/R system. Importantly in strain PUPa3 the levels of C12-3-oxo-HSL were not affected by SICs of tobramycin possibly indicating that the independent organization of the two systems allows them to respond differently to environmental conditions. Another study involving $P$. aeruginosa PAO1, has also reported effects by tobramycin signaling. Linares et al. [11], have reported that SIC of tobramycin induces swarming as well as biofilm formation in P. aeruginosa PAO1. This is contrast of what we report here and what was reported by Luke et al. [36] since we observed a decrease in swarming and biofilm formation when $P$. aeruginosa PUPa3 is exposed to SICs of tobramycin. It is most likely that tobramycin signaling can have different effects in different strains when grown in different growth conditions. In fact another recent study reported that SICs of tobramycin in P. aeruginosa PAO1 did not inhibit AHL QS [12] as we observe for strain PUPa3. Three antibiotics however were reported to inhibit AHL QS at SIC in P. aeruginosa PAO1 [12]; interestingly these antibiotics, namely floroquinolone, cephalosporin and azithromycin, belong to different classes. The inhibition of the RhlI/R system in strain PUPa3 by SIC of tobramycin observed here is similar to what was reported for azithromycin signaling in strain PAO1, however in PAO1 both Las and Rhl systems are inhibited $[18,38,39]$. Interestingly this inhibition is also not due to a decrease in the transcription of the AHL QS systems but apparently to an effect on loci upstream the $l a s I / R$ and $r h l I / R$ systems [38]. It therefore appears that there are some common features between the signaling response of strain PAO1 to azithromycin and tobramycin signaling in strain PUPa3.

How does SIC of tobramycin affect the RhlI/R system? The fact that the LasI/R system was not affected showed that the SIC of tobramycin effect is specific for the RhlI/ $\mathrm{R}$ system. Since a few studies have reported that SIC of several antibiotics affected gene transcription, we 
analyzed whether rhlI transcription levels changed at SICs of tobramycin. The rhlI transcription levels did not change upon exposure to SIC of tobramycin at various growth stages indicating that the lower C4-HSL levels were not due to a decrease of rhlI transcription. It cannot be excluded that this could be due to an effect on rhlI translation; recently it has been reported that a novel protein designated GidA, post-transcriptionally controls RhlI/R levels [40]. In addition, tobramycin at subinhibitory concentrations could result in lower pools of the precursors necessary for C4-HSL biosynthesis or possibly the transport of the AHL could also be affected. The C4-HSL however has been shown that when added to cell suspensions of $P$. aeruginosa, the cellular concentration reached a steady state in less than 30 seconds being equal to the external concentration, hence it can be regarded as a freely diffusible compound [41]. Future studies need to determine the cellular targets which are affected by subinhibitory concentrations of tobramycin which result in lower C4-HSL levels in $P$. aeruginosa PUPa3. Previous studies in P. aeruginosa PAO1 have identified a gene called arr which is involved in tobramycin signaling at SIC [14] which results in an induction of biofilm formation. This gene encodes for an inner membrane protein and it was postulated to respond to tobramycin and affect surface appendages which are important for biofilm formation. The mode of action of Arr is currently unknown but it is believed to occur via the second messenger c-di-GMP since arr mutants are less active in degrading c-di-GMP [14]. It is currently unknown whether reduction of AHL levels by tobramycin signaling occurs via arr.

The tobramycin signaling reported here requires the interaction of tobramycin with the ribosome since ribosomal protection by methylation using the NpmA, RmtA and $\mathrm{RmtC}$ methyltransferases relieves signaling since SICs of tobramycin no longer affect the RhlI/R system. Tobramycin blocks the peptide exit channel by binding to the A1408 residue of $16 \mathrm{~S}$ rRNA and the three methyltransferases specifically methylate nucleotides A1408 [23] and G1405 [33,34] respectively, within the decoding A site in $16 \mathrm{~S}$ rRNA, no longer allowing tobramycin binding. Interestingly azithromycin signaling at SIC in strain $\mathrm{PAO} 1$ also requires interaction with the ribosome since similar experiments demonstrated that the ErmAB methyltranserase which blocks azithromycin interaction with the $23 \mathrm{~S}$ rRNA stops signalling at SICs [39]. These observations indicate that antibiotic signaling requires interaction with the ribosome possibly meaning the regulatory cascade is initiated at the ribosome.

\section{Conclusions}

In summary this study has further highlighted the presence of antibiotic signaling in $P$. aeruginosa and that different strains can have a different response. The role of antibiotics as signaling molecules now needs to be further evidenced by unraveling the molecular mechanisms of the cascade identifying primary response components which trigger the regulatory cascade. Understanding the tobramycin response by $P$. aeruginosa will have important implications in the mode of utilization for human use of this very important antibiotic.

\section{Acknowledgements}

GMV gratefully acknowledges the support from FP6 (grant \#043682 "EuroPharm" as support to FB), ICGEB (grant CRP/CRO08-02) and Croatian Ministry of Science (grant 006-0982913-1219). We thank Iris Bertani, Giulia Devescovi, Giuliano Degrassi, Miguel Mano and Zulma Suarez-Moreno for helpful suggestions and assistance.

\section{Author details}

${ }^{1}$ Department of Biochemistry and Molecular Biology, Faculty of Pharmacy and Biochemistry, University of Zagreb, Ante Kovačića 1, 10000 Zagreb, Croatia. ${ }^{2}$ Bacteriology Group, International Centre for Genetic Engineering and Biotechnology, Area Science Park, Padriciano 99, 34149 Trieste, Italy.

\section{Authors' contributions}

FB performed all the experimental work and was also involved in manuscript writing. W and GMV were involved in supervision of experimental design and data analysis, coordinated the study and drafted the manuscript. All authors read and approved the manuscript.

\section{Competing interests}

The authors declare that they have no competing interests.

Received: 23 December 2009 Accepted: 2 June 2010

Published: 2 June 2010

\section{References}

1. Borovinskaya MA, Pai RD, Zhang W, Schuwirth BS, Holton JM, Hirokawa G, Kaji H, Kaji A, Cate JHD: Structural basis for aminoglycoside inhibition of bacterial ribosome recycling. Nat Struct Mol Biol 2007, 14(8):727-732.

2. Fourmy D, Recht MI, Blanchard SC, Puglisi JD: Structure of the A Site of Escherichia coli 16 S Ribosomal RNA Complexed with an Aminoglycoside Antibiotic. Science 1996, 274(5291):1367-1371.

3. Smith RS, Iglewski BH: P. aeruginosa quorum-sensing systems and virulence. Current Opinion in Microbiology 2003, 6(1):56-60.

4. Vicens $Q$, Westhof E: Crystal Structure of a Complex between the Aminoglycoside Tobramycin and an Oligonucleotide Containing the Ribosomal Decoding A Site. Chemistry \& Biology 2002, 9(6):747-755.

5. Kotra LP, Haddad J, Mobashery S: Aminoglycosides: Perspectives on Mechanisms of Action and Resistance and Strategies to Counter Resistance. Antimicrob Agents Chemother 2000, 44(12):3249-3256.

6. Davies J: Microbes have the last word. A drastic re-evaluation of antimicrobial treatment is needed to overcome the threat of antibioticresistant bacteria. EMBO reports 2007, 8(7):616-621.

7. Davies J, Spiegelman GB, Yim G: The world of subinhibitory antibiotic concentrations. Current Opinion in Microbiology 2006, 9(5):445-453.

8. Ryan RP, Dow JM: Diffusible signals and interspecies communication in bacteria. Microbiology 2008, 154(7):1845-1858.

9. Yim G, Huimi Wang H, Davies Frs J: Antibiotics as signalling molecules. Philosophical Transactions of the Royal Society B: Biological Sciences 2007, 362(1483):1195-1200.

10. Goh E-B, Yim G, Tsui W, McClure J, Surette MG, Davies J: Transcriptional modulation of bacterial gene expression by subinhibitory concentrations of antibiotics. Proceedings of the National Academy of Sciences of the United States of America 2002, 99(26):17025-17030.

11. Linares JF, Gustafsson I, Baquero F, Martinez JL: Antibiotics as intermicrobial signaling agents instead of weapons. Proceedings of the National Academy of Sciences 2006, 103(51):19484-19489. 
12. Skindersoe ME, Alhede M, Phipps R, Yang L, Jensen PO, Rasmussen TB, Bjarnsholt T, Tolker-Nielsen T, Hoiby N, Givskov M: Effects of Antibiotics on Quorum Sensing in Pseudomonas aeruginosa. Antimicrob Agents Chemother 2008, 52(10):3648-3663.

13. Tanaka M, Hasegawa T, Okamoto A, Torii K, Ohta M: Effect of Antibiotics on Group A Streptococcus Exoprotein Production Analyzed by TwoDimensional Gel Electrophoresis. Antimicrob Agents Chemother 2005, 49(1):88-96.

14. Hoffman $L R$, D'Argenio DA, MacCoss MJ, Zhang Z, Jones RA, Miller SI: Aminoglycoside antibiotics induce bacterial biofilm formation. Nature 2005, 436(7054):1171-1175

15. Juhas M, Eberl L, Tümmler B: Quorum sensing: the power of cooperation in the world of Pseudomonas. Environmental Microbiology 2005, 7(4):459-471.

16. Venturi V: Regulation of quorum sensing in Pseudomonas. FEMS Microbiology Reviews 2006, 30(2):274-291.

17. Nalca Y, Jansch L, Bredenbruch F, Geffers R, Buer J, Haussler S: QuorumSensing Antagonistic Activities of Azithromycin in Pseudomonas aeruginosa PAO1: a Global Approach. Antimicrob Agents Chemother 2006, 50(5):1680-1688

18. Tateda K, Comte R, Pechere J-C, Kohler T, Yamaguchi K, Van Delden C: Azithromycin Inhibits Quorum Sensing in Pseudomonas aeruginosa. Antimicrob Agents Chemother 2001, 45(6):1930-1933.

19. Kumar RS, Ayyadurai N, Pandiaraja P, Reddy AV, Venkateswarlu Y, Prakash O, Sakthivel N: Characterization of antifungal metabolite produced by a new strain $<$ i $>$ Pseudomonas aeruginosa</i> PUPa3 that exhibits broadspectrum antifungal activity and biofertilizing traits. Journal of Applied Microbiology 2005, 98(1):145-154.

20. Hanahan D: Studies on transformation of Escherichia coli with plasmids. J Mol Biol 1983, 166:557-580.

21. Figurski DH, Helinski DR: Replication of an origin-containing derivative of plasmid RK2 dependent on a plasmid function provided in trans. Proceedings of the National Academy of Sciences of the United States of America 1979, 76(4):1648-1652.

22. Sambrook J, Fritsch EF, Maniatis T: Molecular cloning: a laboratory manual. Cold Spring Harbor, NY: Cold Spring Harbor Laboratory Press, 21989.

23. Wachino J-i, Shibayama K, Kurokawa H, Kimura K, Yamane K, Suzuki S, Shibata N, Ike Y, Arakawa Y: Novel Plasmid-Mediated 16 S rRNA m1A1408 Methyltransferase, NpmA, Found in a Clinically Isolated Escherichia coli Strain Resistant to Structurally Diverse Aminoglycosides. Antimicrob Agents Chemother 2007, 51(12):4401-4409.

24. Maravic-Vlahovicek G, Cubrilo S, Tkaczuk KL, Bujnicki JM: Modeling and experimental analyses reveal a two-domain structure and amino acids important for the activity of aminoglycoside resistance methyltransferase Sgm. Biochimica et Biophysica Acta (BBA) - Proteins \& Proteomics 2008, 1784(4):582-590.

25. Bradford $\mathrm{M}$ : A rapid and sensitive method for the quantitation of microgram quantities of protein utilizing the principle of protein-dye binding. Anal Biochem 1976, 72:248-254.

26. Steindler L, Bertani I, De Sordi L, Schwager S, Eberl L, Venturi V: LasI/R and Rhll/R Quorum Sensing in a Strain of Pseudomonas aeruginosa Beneficial to Plants. Appl Environ Microbiol 2009, 75(15):5131-5140.

27. Huber B, Riedel K, Hentzer M, Heydorn A, Gotschlich A, Givskov M, Molin S, Eberl L: The cep quorum-sensing system of Burkholderia cepacia H111 controls biofilm formation and swarming motility. Microbiology 2001, 147(9):2517-2528.

28. Winson MK, Swift S, Fish L, Throup JP, Jørgensen F, Chhabra SR, Bycroft BW, Williams $P$, Stewart $G, S A B$ : Construction and analysis of luxCDABE based plasmid sensors for investigating $\mathrm{N}$-acyl homoserine lactone-mediated quorum sensing. FEMS Microbiology Letters 1998, 163(2):185-192.

29. Swift S, Karlyshev AV, Fish L, Durant EL, Winson MK, Chhabra SR, Williams P, Macintyre S, Stewart GS: Quorum sensing in Aeromonas hydrophila and Aeromonas salmonicida: identification of the LuxRl homologs AhyRl and AsaRl and their cognate $\mathrm{N}$-acylhomoserine lactone signal molecules. J Bacteriol 1997, 179(17):5271-5281.

30. Rampioni G, Polticelli F, Bertani I, Righetti K, Venturi V, Zennaro E, Leoni L: The Pseudomonas Quorum-Sensing Regulator RsaL Belongs to the Tetrahelical Superclass of H-T-H Proteins. J Bacteriol 2007, 189(5):1922-1930.

31. Essar DW, Eberly L, Hadero A, Crawford IP: Identification and characterization of genes for a second anthranilate synthase in
Pseudomonas aeruginosa: interchangeability of the two anthranilate synthases and evolutionary implications. J Bacteriol 1990, 172(2):884-900

32. Brint JM, Ohman DE: Synthesis of multiple exoproducts in Pseudomonas aeruginosa is under the control of RhlR-Rhll, another set of regulators in strain PAO1 with homology to the autoinducer-responsive LuxR-LuxI family. J Bacteriol 1995, 177(24):7155-7163.

33. Wachino J-i, Yamane K, Shibayama K, Kurokawa H, Shibata N, Suzuki S, Doi Y, Kimura K, Ike Y, Arakawa Y: Novel Plasmid-Mediated 16 S rRNA Methylase, RmtC, Found in a Proteus mirabilis Isolate Demonstrating Extraordinary High-Level Resistance against Various Aminoglycosides. Antimicrob Agents Chemother 2006, 50(1):178-184.

34. Yokoyama K, Doi Y, Yamane K, Kurokawa H, Shibata N, Shibayama K, Yagi T, Kato $\mathrm{H}$, Arakawa Y: Acquisition of $16 \mathrm{~S}$ rRNA methylase gene in Pseudomonas aeruginosa. The Lancet 2003, 362(9399):1888-1893.

35. Barclay ML: Adaptive resistance to tobramycin in Pseudomonas aeruginosa lung infection in cystic fibrosis. J Antimicrob Chemother 1996 37:1155-1164.

36. Luke A, Beatson SA, Leech AJ, Walsh SL, Bell SC: Sub-inhibitory concentrations of ceftazidime and tobramycin reduce the quorum sensing signals of Pseudomonas aeruginosa. Pathology 2004, 36:571-575.

37. Latifi A, Foglino M, Tanaka K, Williams P, Lazdunski A: A hierarchical quorum-sensing cascade in Pseudomonas aeruginosa links the transcriptional activators LasR and RhIR (VsmR) to expression of the stationary-phase sigma factor RpoS. Mol Microbiol 1996, 21(6):1137-1146.

38. Kai T, Tateda K, Kimura S, Ishii Y, Ito H, Yoshida H, Kimura T, Yamaguchi K: A low concentration of azithromycin inhibits the mRNA expression of $\mathrm{N}$-acyl homoserine lactone synthesis enzymes, upstream of lasl or rhll, in Pseudomonas aeruginosa. Pulmonary Pharmacology \& Therapeutics 2009, 22(6):483-486.

39. Kohler T, Dumas J-L, Van Delden C: Ribosome Protection Prevents Azithromycin-Mediated Quorum-Sensing Modulation and StationaryPhase Killing of Pseudomonas aeruginosa. Antimicrob Agents Chemother 2007, 51(12):4243-4248

40. Gupta R, Gobble TR, Schuster M: GidA posttranscriptionally regulates rhl quorum sensing in Pseudomonas aeruginosa. J Bacteriol 2009, 191(18):5785-5792

41. Pearson JP, Van Delden C, Iglewski BH: Active efflux and diffusion are involved in transport of Pseudomonas aeruginosa cell-to-cell signals. J Bacteriol 1999, 181(4):1203-1210

42. Kumar RS, Ayyadurai N, Pandiaraja P, Reddy AV, Venkateswarlu Y, Prakash $\mathrm{O}$, Sakthivel N: Characterization of antifungal metabolite produced by a new strain Pseudomonas aeruginosa PUPa3 that exhibits broad-spectrum antifungal activity and biofertilizing traits. J Appl Microbiol 2005, 98(1):145-154.

43. Steindler L, Bertani I, De Sordi L, Schwager S, Eberl L, Venturi V: LasI/R and Rhll/R quorum sensing in a strain of Pseudomonas aeruginosa beneficial to plants. Appl Environ Microbiol 2009, 75(15):5131-5140.

44. Sambrook J, Fritsch EF, Maniatis T: Molecular cloning: a laboratory manual. Cold Spring Harbor, N.Y., 21989.

45. Spaink HP, Okker RJH, Wijffelmann CA, Pees E, Lugtemberg BJJ: Promoter in the nodulation region of the Rhizobium leguminosarum Sym plasmid pRL1Jl. Plant Mol Biol 1987, 9:27-39.

46. Kovach ME, Elzer PH, Hill DS, Robertson GT, Farris MA, Roop RM, Peterson KM: Four new derivatives of the broad-host-range cloning vector $\mathrm{pBBR} 1 \mathrm{MCS}$, carrying different antibiotic-resistance cassettes. Gene 1995, 166(1):175-176.

47. Bertani I, Venturi V: Regulation of the N-Acyl Homoserine LactoneDependent Quorum-Sensing System in Rhizosphere Pseudomonas putida WCS358 and Cross-Talk with the Stationary-Phase RpoS Sigma Factor and the Global Regulator GacA. Appl Environ Microbiol 2004, 70(9):5493-5502.

48. Winson MK, Swift S, Fish L, Throup JP, Jorgensen F, Chhabra SR, Bycroft BW, Williams P, Stewart GS: Construction and analysis of luxCDABE-based plasmid sensors for investigating $\mathrm{N}$-acyl homoserine lactone-mediated quorum sensing. FEMS Microbiol Lett 1998, 163(2):185-192.

49. Swift S, Karlyshev AV, Fish L, Durant EL, Winson MK, Chhabra SR, Williams P, Macintyre S, Stewart GS: Quorum sensing in Aeromonas hydrophila and Aeromonas salmonicida: identification of the LuxRI homologs AhyRI and AsaRl and their cognate $\mathrm{N}$-acylhomoserine lactone signal molecules. J Bacteriol 1997, 179(17):5271-5281. 
50. Figurski DH, Helinski DR: Replication of an origin-containing derivative of plasmid RK2 dependent on a plasmid function provided in trans. Proc Natl Acad Sci USA 1979, 76(4):1648-1652.

51. Kojic M, Venturi V: Regulation of rpoS gene expression in Pseudomonas: involvement of a TetR family regulator. J Bacteriol 2001, 183(12):3712-3720.

52. Rampioni G, Bertani I, Zennaro E, Polticelli F, Venturi V, Leoni L: The quorum-sensing negative regulator RsaL of Pseudomonas aeruginosa binds to the lasl promoter. J Bacteriol 2006, 188(2):815-819.

\section{Pre-publication history}

The pre-publication history for this paper can be accessed here: http://www.biomedcentral.com/1471-2334/10/148/prepub

doi:10.1186/1471-2334-10-148

Cite this article as: Babić et al:: Tobramycin at subinhibitory concentration inhibits the Rhll/R quorum sensing system in a Pseudomonas aeruginosa environmental isolate. BMC Infectious Diseases 2010 10:148

\section{Submit your next manuscript to BioMed Central} and take full advantage of:

- Convenient online submission

- Thorough peer review

- No space constraints or color figure charges

- Immediate publication on acceptance

- Inclusion in PubMed, CAS, Scopus and Google Scholar

- Research which is freely available for redistribution

Submit your manuscript at www.biomedcentral.com/submit 